\title{
Integrated Analysis of Comparative Lipidomics and Proteomics \\ Reveals the Dynamic Changes of Lipid Molecular Species in \\ High-oleic Peanut Seed
}

\section{Authors}

Hao Liu ${ }^{1 \#}$, Yanbin Hong ${ }^{1 \#}$,Qing $\mathrm{Lu}^{1}$, Haifen $\mathrm{Li}^{1}$, Jianzhong $\mathrm{Gu}^{2}$, Li Ren ${ }^{2}$, Li Deng $^{2}$,Baojin Zhou ${ }^{3}$, Xiaoping Chen ${ }^{1 *}$, Xuanqiang Liang ${ }^{1 *}$

\# These authors contributed equally to this work.

* corresponding author.

\section{Affiliations}

${ }^{1}$ Crops Research Institute, Guangdong Academy of Agricultural Sciences, Guangdong Provincial Key Laboratory of Crop Genetic Improvement, South China Peanut Sub-Center of National Center of Oilseed Crops Improvement, Guangzhou, 510640, China.

${ }^{2}$ Peanut Research Institute, Kaifeng Academy of Agriculture and Forestry, Kaifeng 475004, China.

${ }^{3}$ Shenzhen Deepxomics Biotechnology Co. Ltd, Shenzhen 518000, China.

\section{*Corresponding author}

Xiaoping Chen,

E-mail: chenxiaoping@gdaas.cn

Xuanqiang Liang,

E-mail: liangxuanqiang@gdaas.cn

\section{Email addresses}


liuhao2054@stu.scau.edu.cn (HL),

hongyanbin@gdaas.cn (YH),

luqing@gdaas.cn (QL),

lihaifen@gdaas.cn (HFL),

xinkeyan@126.com (JG),

renli120@sina.com(LR),

dengli_1225@sina.com(LD),

zhoubaojin@deepxomics.com (BZ),

chenxiaoping@gdaas.cn(XC),

liangxuanqiang@gdaas.cn (XL). 
A

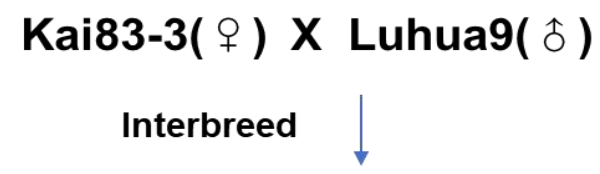

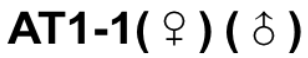

High Oleic

Interbreed

Inbreed

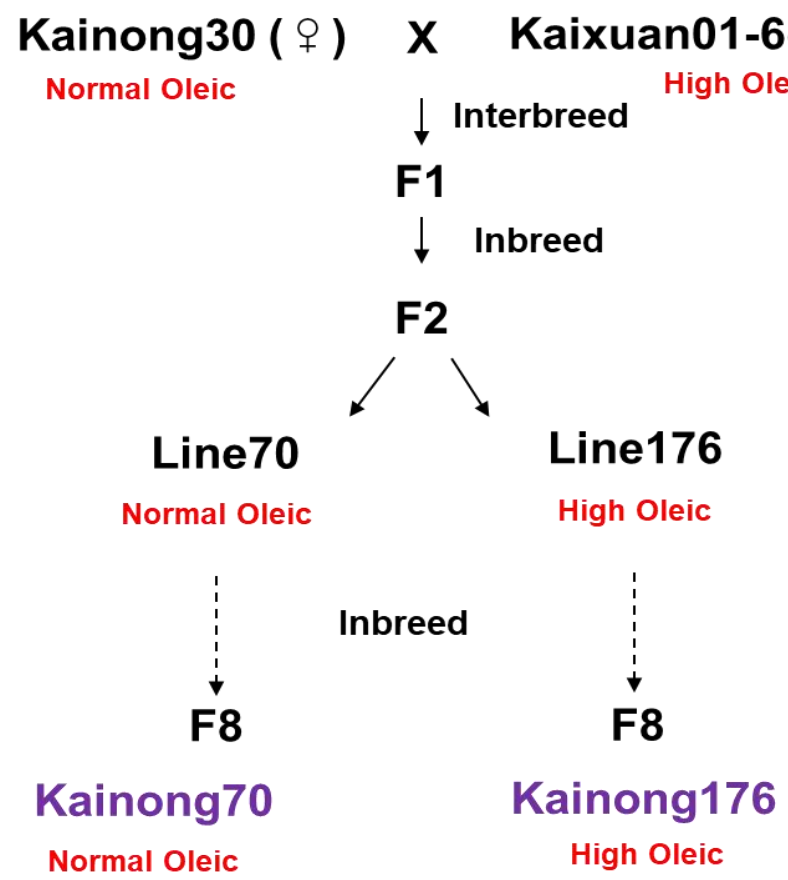


B

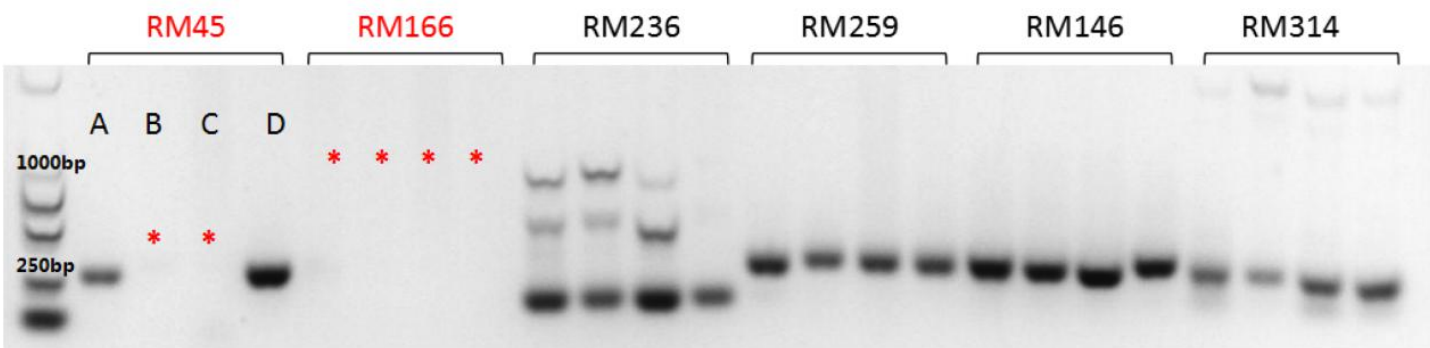

DNA template

A: Kaixuan01-6; B: Kainong30; C: Kainong70 (L70); D: Kainong176 (H176).

* indicates the no amplification.
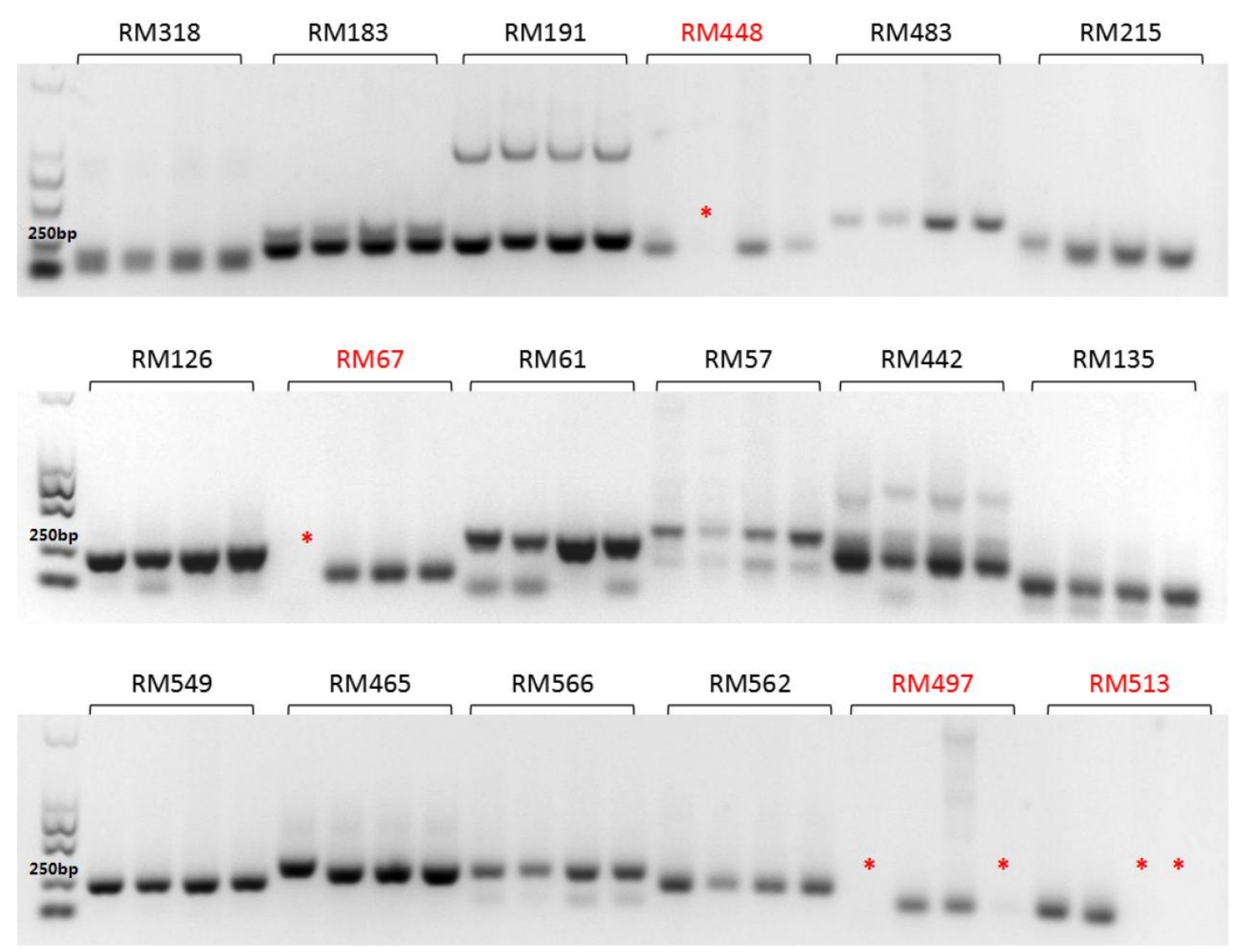


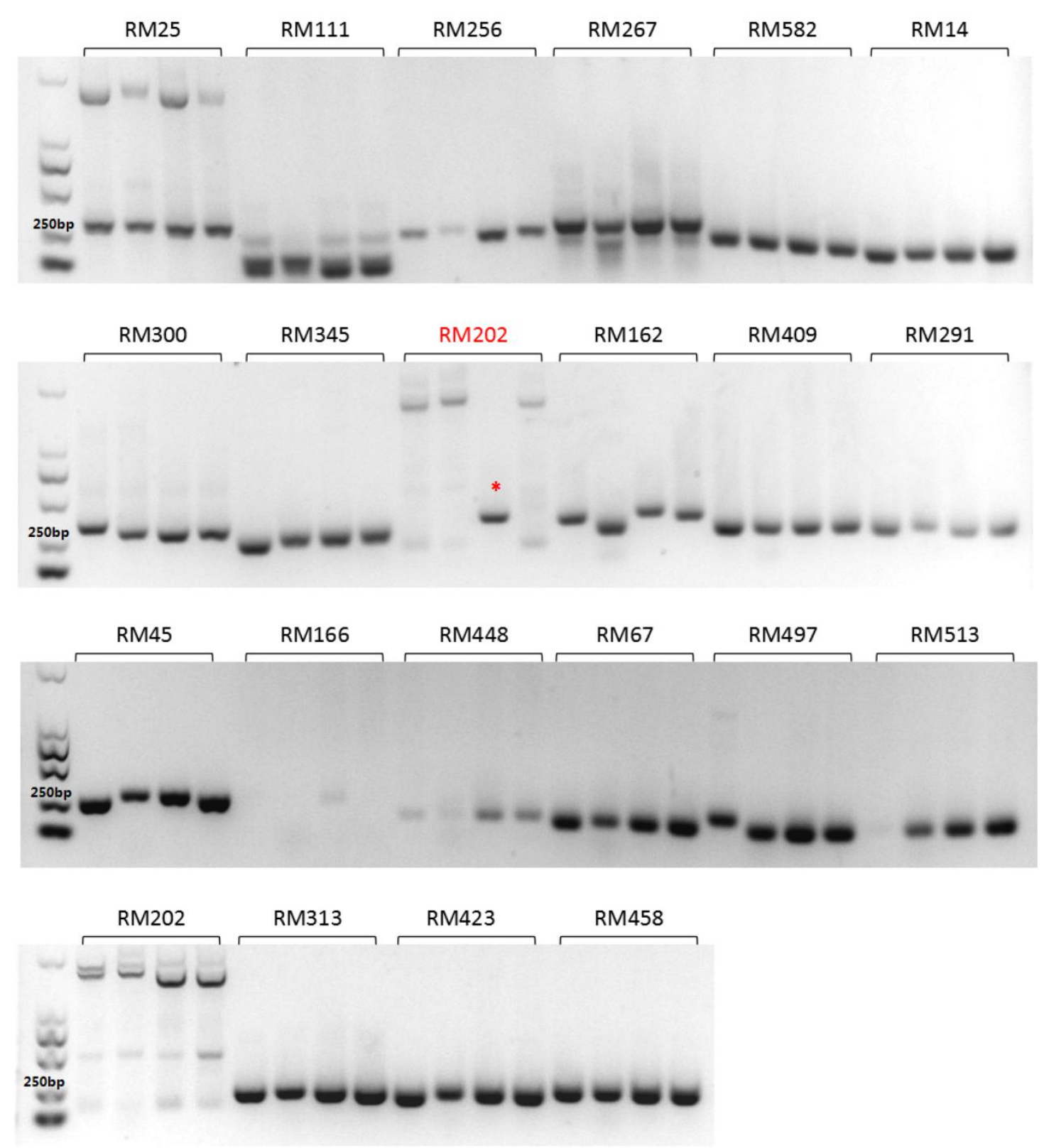




\begin{tabular}{|c|c|c|c|}
\hline Name & Chr. & F primer & $\mathrm{R}$ primer \\
\hline RM61 & $\mathrm{a} 01$ & GGAGAACCAGTGACGTGACATA & GGATTAATTCTGATACCATGAAAGG \\
\hline RM67 & $\mathrm{a} 01$ & TCAGGGTGGGTTACCAACAT & TAATCCACATTGAACCGACG \\
\hline RM126 & $\mathrm{a} 02$ & GATCTTTCCGCCATTTTCTC & GGTGAATGACAGATGCTCCA \\
\hline RM166 & $\mathrm{a} 03$ & TTCGGCTGACAGCTCTAAG & GAAAGAAATTATACACTCCAATTATGC \\
\hline RM45 & $\mathrm{a} 03$ & AATGTGGCGTTCTTCTTCGT & TCCTATCCGTTATCCCCCTC \\
\hline RM259 & a04 & ACAATGCAATGACCGTTGTT & TTGTTGCATGAGAACGTGAA \\
\hline RM236 & a04 & CAGCGGCAACAGTTTTGATG & GAAAAGTATGCCGCCGTTG \\
\hline RM146 & $\mathrm{a} 05$ & TATTTGTTTCTGGTTCCGCC & TCTGTTAGAGAAGTCAAGTCTGTTG \\
\hline RM314 & a05 & CAGGCTTAAACTCCGTGAGC & ACTTGGATGACCCGGTACAA \\
\hline RM318 & a06 & GGCAGGGGAATAAAACTACTAACT & TTTTCстTсстTстсстTTGTC \\
\hline RM183 & a06 & ATGACAAGGCTCTTTCGATCC & TACTTGACCCTCCTCCTCCAT \\
\hline RM448 & $\mathrm{a} 07$ & TCCTTCCCACAATAACAATGAA & GAGGAGAAAACATGGCCTAAAA \\
\hline RM191 & $\mathrm{a} 07$ & TGCTTTTGCTCAACATGCTA & ACAGACATTGCAGCTTCACG \\
\hline RM483 & a08 & GGGAATAGCGAGATACATGTCAG & CAGGAGAGAAGGATTGTGCC \\
\hline RM215 & $\mathrm{a} 08$ & TGTCCGAACAACTTGAGACG & GCGTGGTCATACACACTTGG \\
\hline RM267 & a09 & ACGTCAGGTTCACAACGACA & ATTTTTCTCCGAGTCAGCCA \\
\hline RM256 & a09 & CAATCAAATCAATAATGTCTCTTTCTC & CGCTCGACCAAGAAAAGTTC \\
\hline RM442 & $\mathrm{a} 10$ & TTCGGTCATGTTTGTCCAGA & CTCGAGTGCTCACCCTTCAT \\
\hline RM582 & $\mathrm{a} 10$ & TGAGGCCGTCTTGTTTAGAGA & ССТCTTCCATCACCGTTCATA \\
\hline RM300 & b01 & TACTACTGTTGCCGCTGTGC & TTTCTCACCATTGGCATTCA \\
\hline RM111 & b01 & СТCTCTCTCCCCTTCATCGAC & GGCAGAATGAAAGGTGAGAACT \\
\hline RM135 & b02 & CCTCACTTCTTTTTGCATGGT & TGGAAAGGAAATGATTTGGTG \\
\hline RM345 & $\mathrm{b} 02$ & GCTAAGCTAAATTACCCATTTTGTG & GTTTGAGCTTGTGCAGTGGA \\
\hline RM162 & $\mathrm{b} 03$ & TGTTGCCCACTGTTCTAATCA & TCAAATGGCATAGTCTCCCC \\
\hline RM202 & b03 & CGTTGGGGACAAAAACGATA & TTTTCTTGAAACTCGTTGATATGG \\
\hline RM291 & b04 & CCTCCGTTGCTCTTCTGAAC & GATCAAGCACTTCAGACAATGG \\
\hline RM14 & b04 & CCATGTGAGGTATCAGTAAAGAAAGG & CCACCAACAACATTGGATGAAT \\
\hline RM423 & b05 & CTGCATAGTGGCGGTGATAA & CATCGGATTAATTCAACGGT \\
\hline RM313 & b05 & GCCCATATCAAGCTCCAAAA & TAGCCAGCGAAGGACTCAAT \\
\hline RM409 & b06 & CCGCAGATCTTCTCCTGTGT & CСТCСТCATCCTCTAAACTCTGC \\
\hline RM458 & b06 & ATTCCGCGAACTTCATTAGC & GGATTGAATGGCAAAGAGGA \\
\hline RM25 & b07 & GCCGAGCTAGTTTGATTTGG & TTGGATTTGAATGGAGGAATG \\
\hline RM465 & b07 & TTAGCGACAAAGGATGGTGAG & TAGGGACGAAAATAGGGACTGA \\
\hline RM497 & b08 & GTTGTGGTGACGAGCTCAAA & CCACTAACCACCCCATCATC \\
\hline RM513 & b08 & TGGGAGTCATGGCAATTTTT & CACCATCTCTCATCCATTTTCA \\
\hline RM562 & b09 & TCGGTTTGGGAGACACTCTT & TTGTAAGCAGACGCCACATC \\
\hline RM566 & b09 & CAAGCATCAACAACAACGA & GTCCGACCACATACAAGAGTT \\
\hline RM57 & b10 & TATTTCTGCTTCGGCGCTAT & TTTCCCCCATCTCACTCAAC \\
\hline RM549 & b10 & AATACССТTCССCAATCACC & TGCTTCTGCTCGATGTTCTG \\
\hline
\end{tabular}

Figure S1. The breeding processes of normal- and high- OA cultivars Kainong70 and Kainong176. A, Kai83-3() intercrossed with Luhua9(ô) to cultivate the Kainong30, Kaixuan01-6 selected from the inbreeding population of American introduced high OA peanut variety AT1-1(†) (ð). Kainong30 (†) intercrossed with Kaixuan01-6 (す) to generate the F2 population, normal- and high OA line 70 and 176 isolated from the F2 population, and self-fertilized to generate the normal cultivar Kainong70 and high OA cultivar Kainong176 until the stable F8 population. Information of these varieties was provided by the website of www.peanutdata.cn. B, 39 pairs of SSR markers were 
used to detect the polymorphism of genetic background between the L70, H176 and their parents (kaixuan01-6 and Kainong30). This result indicated that the genetic background of L70 and H176 was very similar, only the RM45 and RM146 performed difference amplification in L70 and H176 genome. Five pairs of SSR marker existed difference in Kaixuan01-6 and Kainong30, including the RM45, RM497, RM300, RM345, and RM162. DNA amplification was observed by agarose gel electrophoresis. 
$>$ FAD2-A coding sequence in high OA cultivar Kainong176

ATGGGAGCTGGAGGGCGTGTCACTAAGATTGAAGCTCAAAAGAAGCCTCT TTCAAGGGTTCCACATTCAAACCCTCCATTCAGTGTTGGCCAACTCAAGAA AGCAATTCCACCACATTGCTTTGAACGTTCTCTTTTCATATCATTCTCCTATG TTGTCTATGATCTCTTAGTGGCCTACTTACTCTTCTACATTGCCACCACTTAT TTCCACAAGCTTCCATACCCATTTTCCTTCCTTGCTTGGCCAATCTATTGGGC CATCCAAGGCTGCATTCTCACTGGTGTTTGGGTGATTGCTCATGAGTGTGG CCACCATGCCTTCAGCAAGTACCAACTTGTTGATGACATGGTTGGTTTGAC CCTTCACTCTTGA(375bp)CTATTAGTTCCTTATTTCTCATGGAAAATCAGCCA CCGCCGCCACCACTCCAACACCGGTTCCCTCGACCGCAACGAAGTGTTTG TCCCAAAACCAAAATCAAAGGTATCATGGTATAACAAGTACATGAACAATC CACCAGGGAGGGCTATCTCCCTCTTCATCACACTCACACTAGGATGGCCCT TGTACTTGGCCTTCAATGTTTCTGGCAGACCCTATGATAGATTTGCAAGCCA CTATGACCCTTATGCTCCCATATACTCTAACAGGGAAAGGCTTCTAATTTATG TCTCAGATTCATCTGTCTTTGCTGTAACATATCTGCTATATCACATAGCAACT TTGAAAGGTTTGGGTTGGGTGGTATGTGTTTATGGGGTGCCATTGCTCATTG TGAATGGGTTTCTAGTTACCATAACCTATTTGCAGCACACACATGCATCATT GACTCACTATGATTCATCCGAATGGGACTGGTTAAGAGGAGCATTGGCAAC AGTGGACAGAGATTATGGGATACTGAATAAGGCATTTCATCATATAACTGAT ACGCATGTGGCTCATCATTTGTTCTCAACAATGCCTCATTACCATGCAATGG AAGCAACCAATGCAATAAAGCCAATATTGGGTGATTACTACCAATTTGATGG CACCCCAGTTTACAAAGCATTGTGGAGAGAAGCCAAAGAGTGCCTCTATGT GGAGCCAGATGATGGAGCTTCTCAGAAGGGTGTTTATTGGTACAAGAACA AGTTCTGA

\section{$>$ FAD2-A in normal OA cultivar Kainong70}

ATGGGAGCTGGAGGGCGTGTCACTAAGATTGAAGCTCAAAAGAAGCCTCT TTCAAGGGTTCCACATTCAAACCCTCCATTCAGTGTTGGCCAACTCAAGAA AGCAATTCCACCACATTGCTTTGAACGTTCTCTTTTCATATCATTCTCCTATG TTGTCTATGATCTCTTAGTGGCCTACTTACTCTTCTACATTGCCACCACTTAT TTCCACAAGCTTCCATACCCATTTTCCTTCCTTGCTTGGCCAATCTATTGGGC CATCCAAGGCTGCATTCTCACTGGTGTTTGGGTGATTGCTCATGAGTGTGG CCACCATGCCTTCAGCAAGTACCAACTTGTTGATGACATGGTTGGTTTGAC CCTTCACTCTTGTCTATTAGTTCCTTATTTCTCATGGAAAATCAGCCACCGCC GCCACCACTCCAACACCGGTTCCCTCGACCGCGACGAAGTGTTTGTCCCA AAACCAAAATCAAAGGTATCATGGTATAACAAGTACATGAACAATCCACCA GGGAGGGCTATCTCCCTCTTCATCACACTCACACTAGGATGGCCCTTGTACT TGGCCTTCAATGTTTCTGGCAGACCCTATGATAGATTTGCAAGCCACTATGA CCCTTATGCTCCCATATACTCTAACAGGGAAAGGCTTCTAATTTATGTCTCAG ATTCATCTGTCTTTGCTGTAACATATCTGCTATATCACATAGCAACTCTGAAA GGTTTGGGTTGGGTGGTATGTGTTTATGGGGTGCCATTGCTCATTGTGAATG GGTTTCTAGTTACCATAACCTATTTGCAGCACACACATGCATCATTGCCTCA CTATGATTCATCCGAATGGGACTGGTTAAGAGGAGCATTGGCAACAGTGGA 
CAGAGATTATGGGATACTGAATAAGGCATTTCATCATATAACTGATACGCATG TGGCTCATCATTTGTTCTCAACAATGCCTCATTACCATGCAATGGAAGCAAC CAATGCAATAAAGCCAATATTGGGTGATTACTACCAATTTGATGGCACCCCA GTTTACAAAGCATTGTGGAGAGAAGCCAAAGAGTGCCTCTATGTGGAGCC AGATGATGGAGCTTCTCAGAAGGGTGTTTATTGGTACAAGAACAAGTTCTG A

$>F A D 2-B$ coding sequence in Kainong176

ATGGGAGCTGGAGGGCGTGTCACTAAGATTGAAGCTCAAAAGAAGCCTCC TTCAAGGGTTCCACATTCAAACCCTCCATTCAGTGTTGGCCAACTCAAGAA GGCAATTCCACCACATTGCTTTGAACGTTCTCTTTTCATATCATTCTCATATG TTGTCTATGATCTCTTAATGGCCTACTTACTCTTCTACATTGCCACCACTTATT TCCACAAGCTTCCATACTCATTATCCTTCCTTGCTTGGCCAATCTATTGGGCC ATCCAAGGCTGCATTCTCACCGGTGTTTGGGTGATTGCTCATGAGTGTGGC CACCATGCCTTCAGCAAGTACCAACTTGTTGATGACATGGTTGGTTTGACC CTTCACTCTTGTCTATTAGTTCCTTATTTCTCGTGGAAAATCAGCCACCGCC GCCACCACTCCAACACAGGTTCCCTCA(442bp)GACCGCGACGAAGTGTTTG TCCCGAAACCAAAATCAAAGGTATCATGGTATAACAAGTACATGAACAATC CACCAGGGAGGGCTATTTCCCTTTTCATCACACCCACACTAGGATGGCCCT TGTACTTGGCCTTCAATGTTTCTGGCAGACCCTATGATAGATTTGCAAGCCA CTATGACCCTTATGCTCCCATATACTCTAACAGGGAAAGGCTTCTAATTTATG TCTCAGATTCATCTGTCTTTGCTGTAACATATCTGCTATATCACATAGCAACT TTGAAAGGTTTGGGTTGGGTGGTATGTGTTTATGGGGTGCCATTGCTCATTG TGAATGGGTTTCTAGTTACCATAACCTATTTGCAGCACACACATGCAGCATT GCCTCACTATGATTCATCCGAATGGGACTGGTTAAGAGGAGCATTGGCAAC AGTGGACAGAGATTATGGGATACTGAATAAGGCATTTCATCATATAACTGAT ACGCATGTGGCTCATCATTTGTTCTCAACAATGCCTCATTACCATGCAATGG AAGCAACCAATGCAATAAAGCCAATATTGGGTGATTACTACCAATTTGATGG CACCCCAGTTTACAAAGCATTGTGGAGAGAAGCCAAAGAGTGCCTCTATGT GGAGCCAGATGATGGAGCTTCTCAGAAGGGTGTTTATTGGTACAAGAACA AGTTCTGA

$>$ FAD2-B coding sequence in Kainong70

ATGGGAGCTGGAGGGCGTGTCACTAAGATTGAAGCTCAAAAGAAGCCTCT TTCAAGGGTTCCACATTCAAACCCTCCATTCAGTGTTGGCCAACTCAAGAA AGCAATTCCACCACATTGCTTTGAACGTTCTCTTTTCATATCATTCTCATATG TTGTCTATGATCTCTTAATGGCCTACTTACTCTTCTACATTGCCACCACTTATT TCCACAAGCTTCCATACCCATTTTCCTTCCTTGCTTGGCCAATCTATTGGGC CATCCAAGGCTGCATTCTCACCGGTGTTTGGGTGATTGCTCATGAGTGTGG CCACCATGCCTTCAGCAAGTACCAACTTGTTGATGACATGGTTGGTTTGAC CCTTCACTCTTGTCTATTAGTTCCTTATTTCTCATGGAAAATCAGCCACCGCC GCCACCACTCCAACACAGGTTCCCTCGACCGCGACGAAGTGTTTGTCCCG 


\begin{abstract}
AAACCAAAATCAAAGGTATCATGGTATAACAAGTACATGAACAATCCACCA GGGAGGGCTATTTCTCTTTTCATCACACTCACACTAGGATGGCCCTTGTACC TGGCCTTCAATGTTTCTGGCAGACCCTATGATAGATTTGCAAGCCACTATGA CCCTTATGCTCCCATATACTCTAACAGGGAAAGGCTTCTAATTTATGTCTCAG ATTCATCTGTCTTTGCTGTAACATATCTGCTATATCACATAGCAACTTTGAAA GGTTTGGGTTGGGTGGTATGTGTTTATGGGGTGCCATTGCTCATTGTGAATG GGTTTCTAGTTACCATAACCTATTTGCAGCACACACATGCATCATTGCCTCA CTATGATTCATCCGAATGGGACTGGTTAAGAGGAGCATTGGCAACAGTGGA CAGAGATTATGGGATACTGAATAAGGCATTTCATCATATAACTGATACGCATG TGGCTCATCATTTGTTCTCAACGATGCCTCATTACCATGCAATGGAAGCAAC CAATGCAATAAAGCCAATATTGGGTGATTACTACCAATTTGATGGCACCCCA GTTTACAAAGCATTGTGGAGAGAAGCCAAAGAGTGCCTCTATGTGGAGCC AGATGATGGAGCTTCTCAGAAGGGTGTTTATTGGTACAAGAACAAGTTCTG A
\end{abstract}

Figure S2. Coding sequence of FAD2-A and FAD2-B in Kainong176 (H176) and Kainong70 (L70). 
A

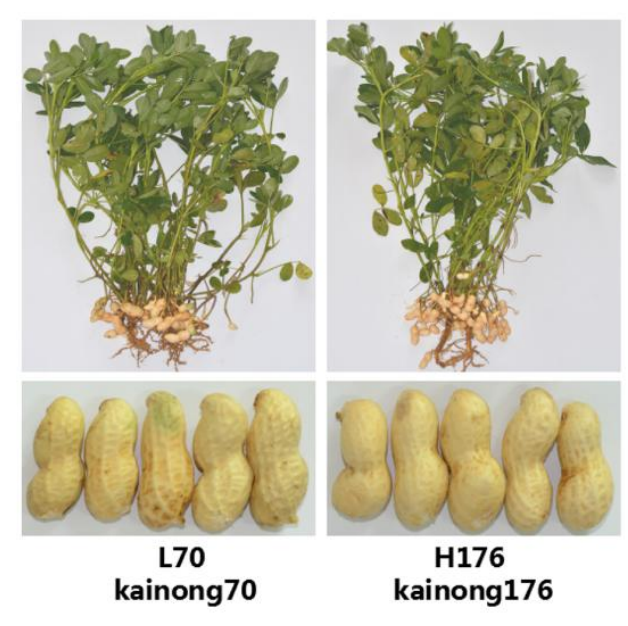

B

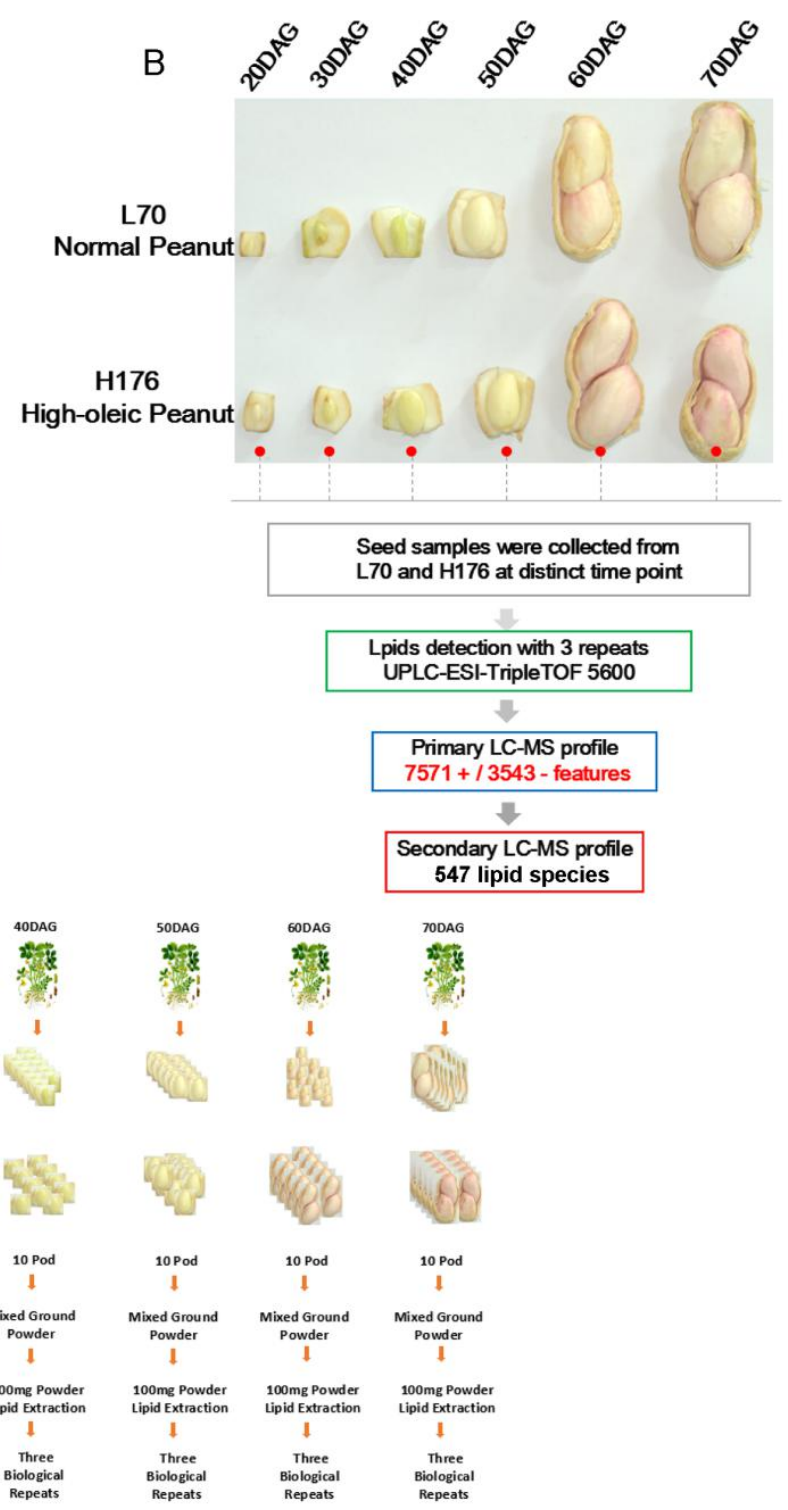

Figure S3. Morphological of normal- (L70, Kainong70) and high- OA (H176, Kainong176) peanut cultivars. A, Phenotypes of L70 and H176 plants. B, Seed samples obtained from six different developmental stages (20-70 DAG) in L70 and H176, respectively. C, The detailed process of lipid sample collection. Each sample contained three biological repeats, and the peanut sample collected from individual plant at each stage. 10 pods were collected from each tree at each stage, and then the seeds were mixed to extract the total lipid. DAG represented the days after gynophores. 

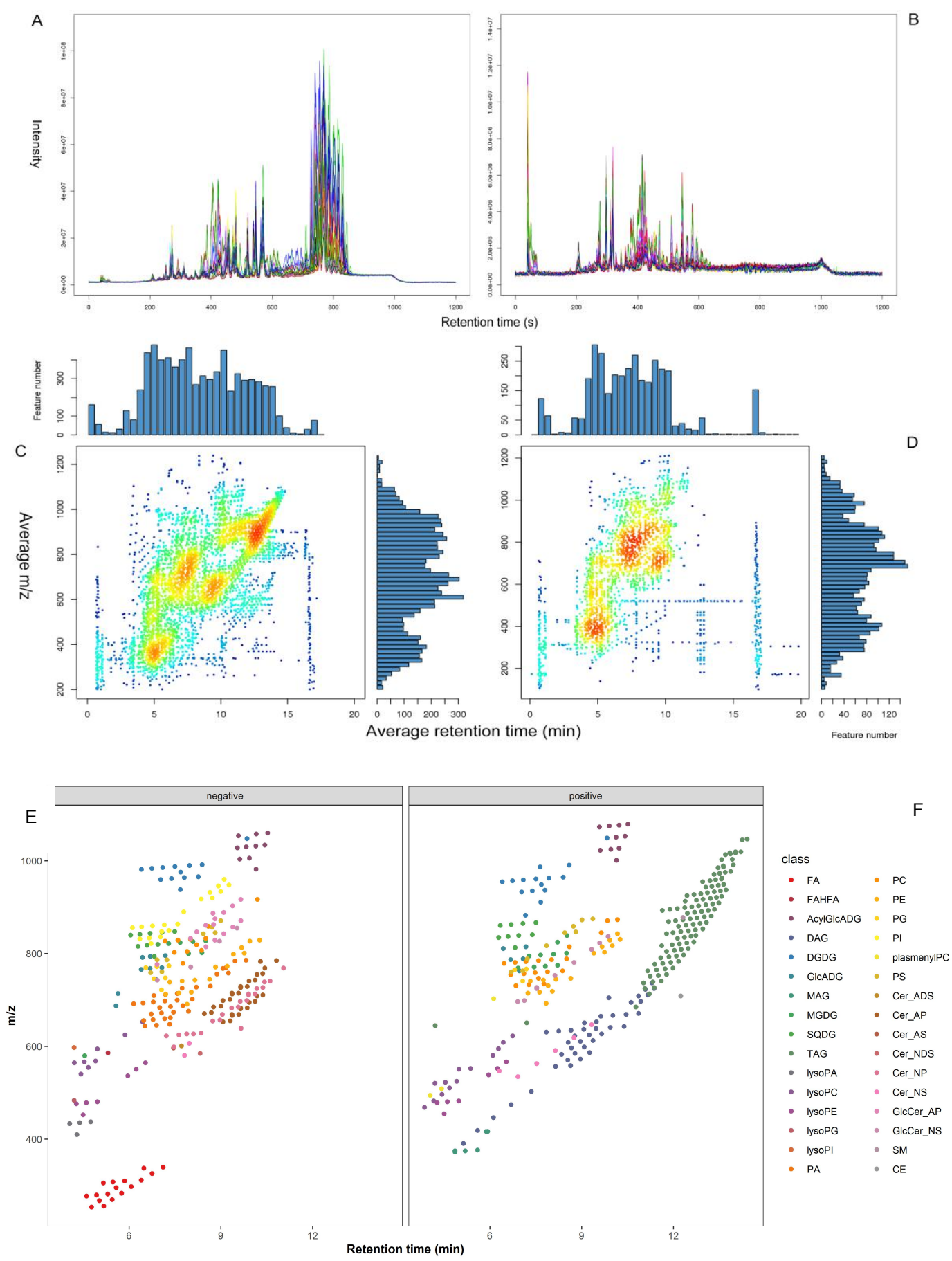

Figure S4. LC-MS identified the lipid features in peanut seed. A-B, LC-MS scan of total ion chromotogram in peanut seed lipid profile under the positive and negative modes, respectively. C-D, Primary LC retention map of total extracted lipid molecular species under the positive and negative scan modes. Y-axis indicated the average $\mathrm{m} / \mathrm{z}$ of each lipid feature, and X-axis indicated the average RT (retention time, min) of each lipid feature. Numerical value indicated the identical lipid feature number, each 
dot represented an individual lipid species, and different colors represented the distribute-intensive situation. E-F, Secondary MS map of total identified lipid classes under the filter pattern with positive and negative modes based on retention time screening, respectively. 

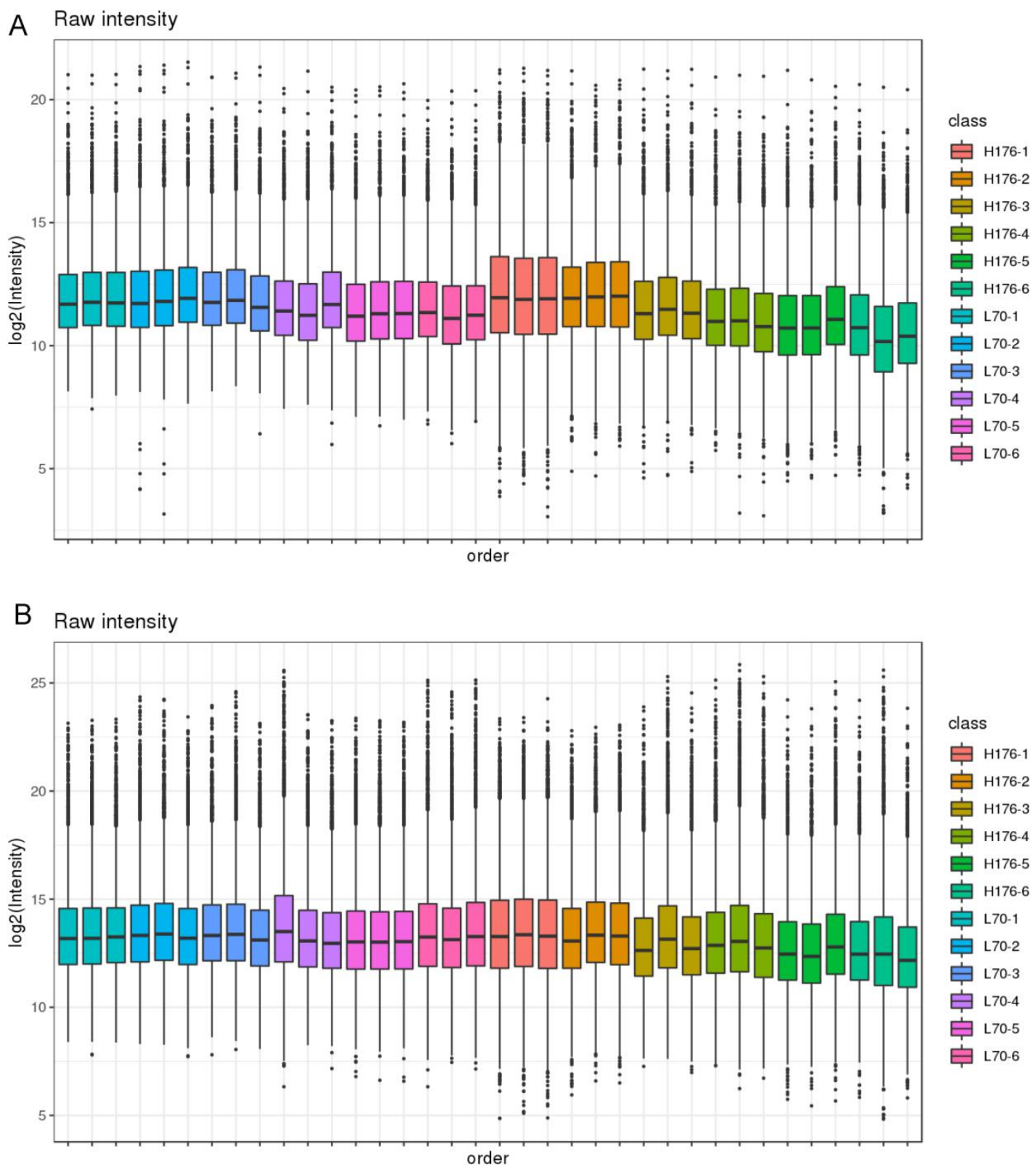

Figure S5. Data normalization analysis of all generated lipidomic datasets under the negative and positive modes, respectively. 
A

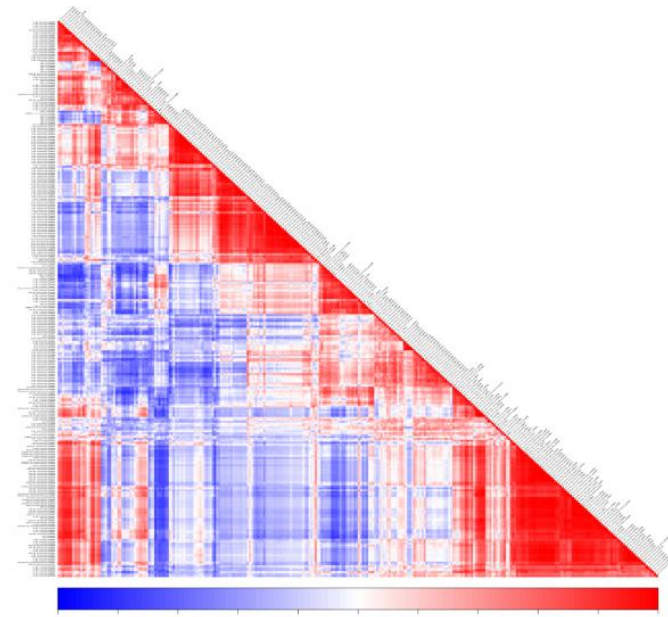

B

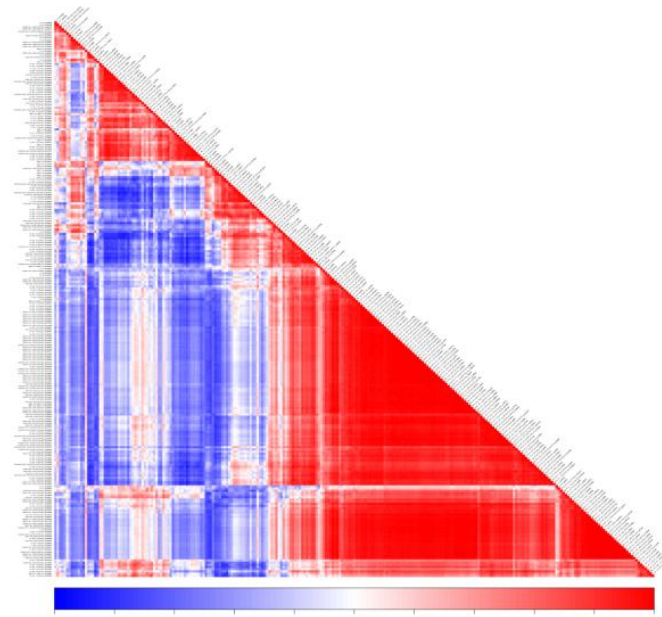

Figure S6. Data correlation analysis of lipid species in tandem mass spectrum profile (MS2). A, Data correlation analysis of all identified lipid molecular species in the positive profile. B, Data correlation analysis of all identified lipid molecular species in the negative profile. 


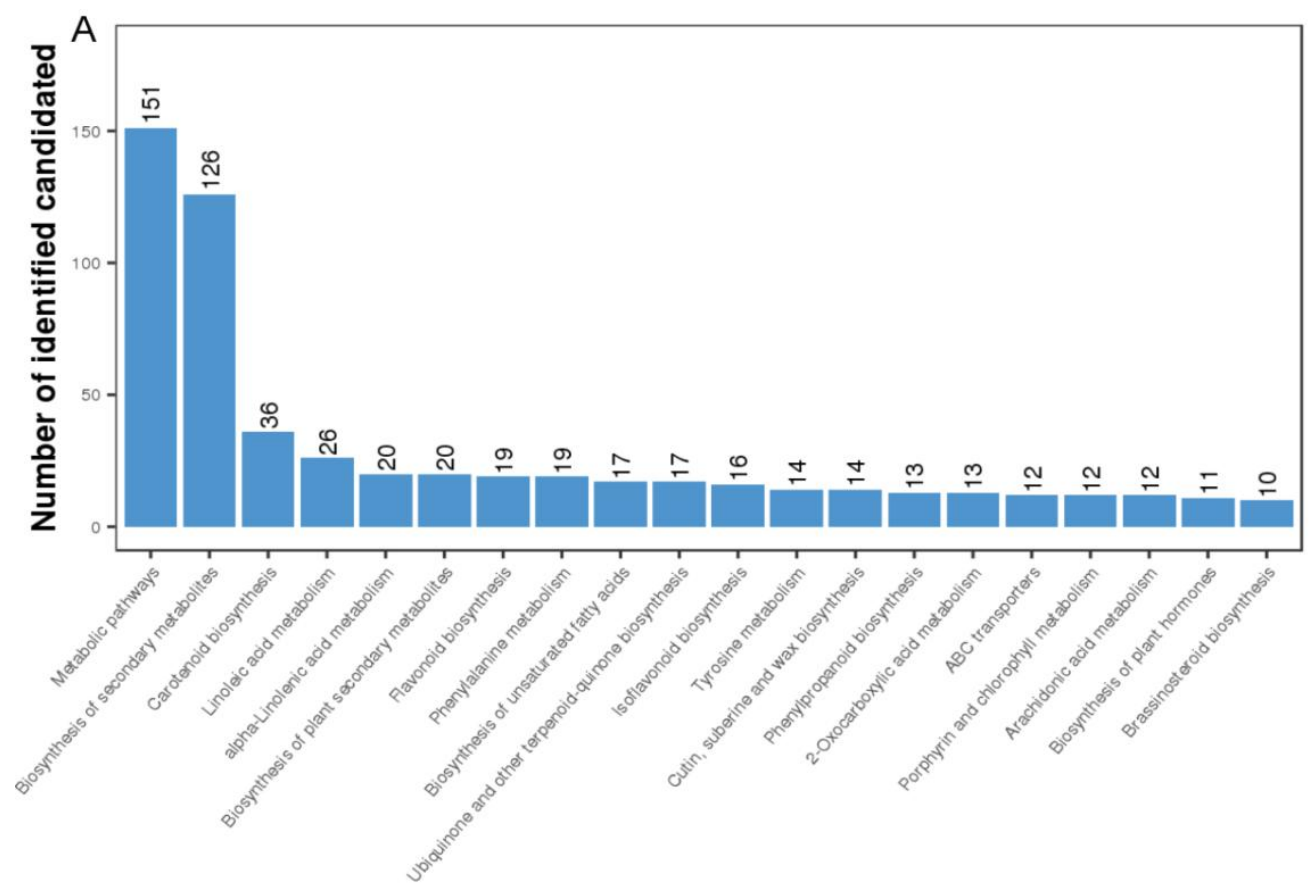

B

Top 20 KEGG pathway

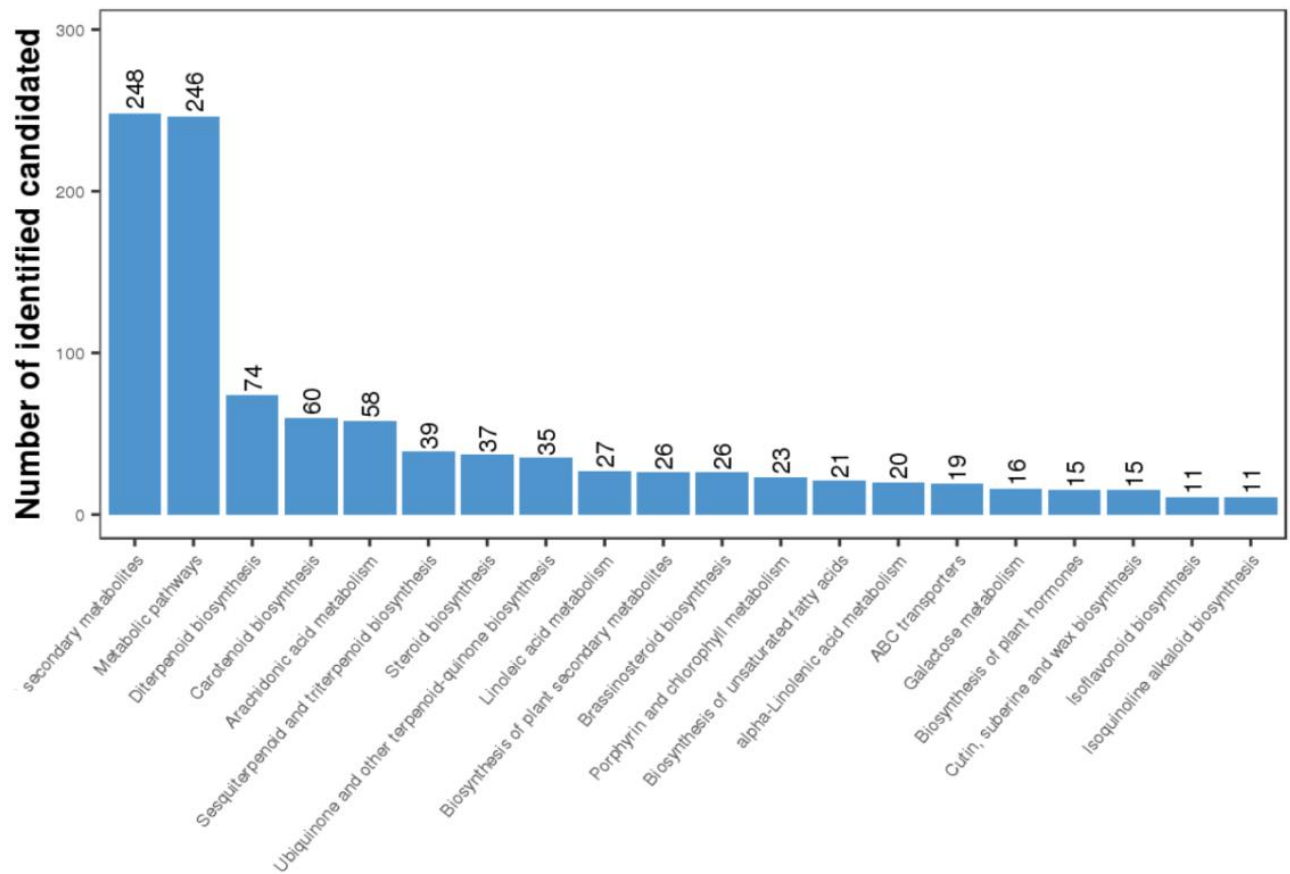

Top 20 KEGG pathway

Figure S7. KEGG pathway enrichment analysis of all identified lipid features in primary mass spectrum profile (top 20 terms). A, KEGG enrichment of all lipid features with negative model. B, KEGG enrichment pathway of all lipid features with positive model. KEGG pathways fulfilling the criterion of a cut-off P-value $\leq 0.05$ were defined as significantly enriched. 


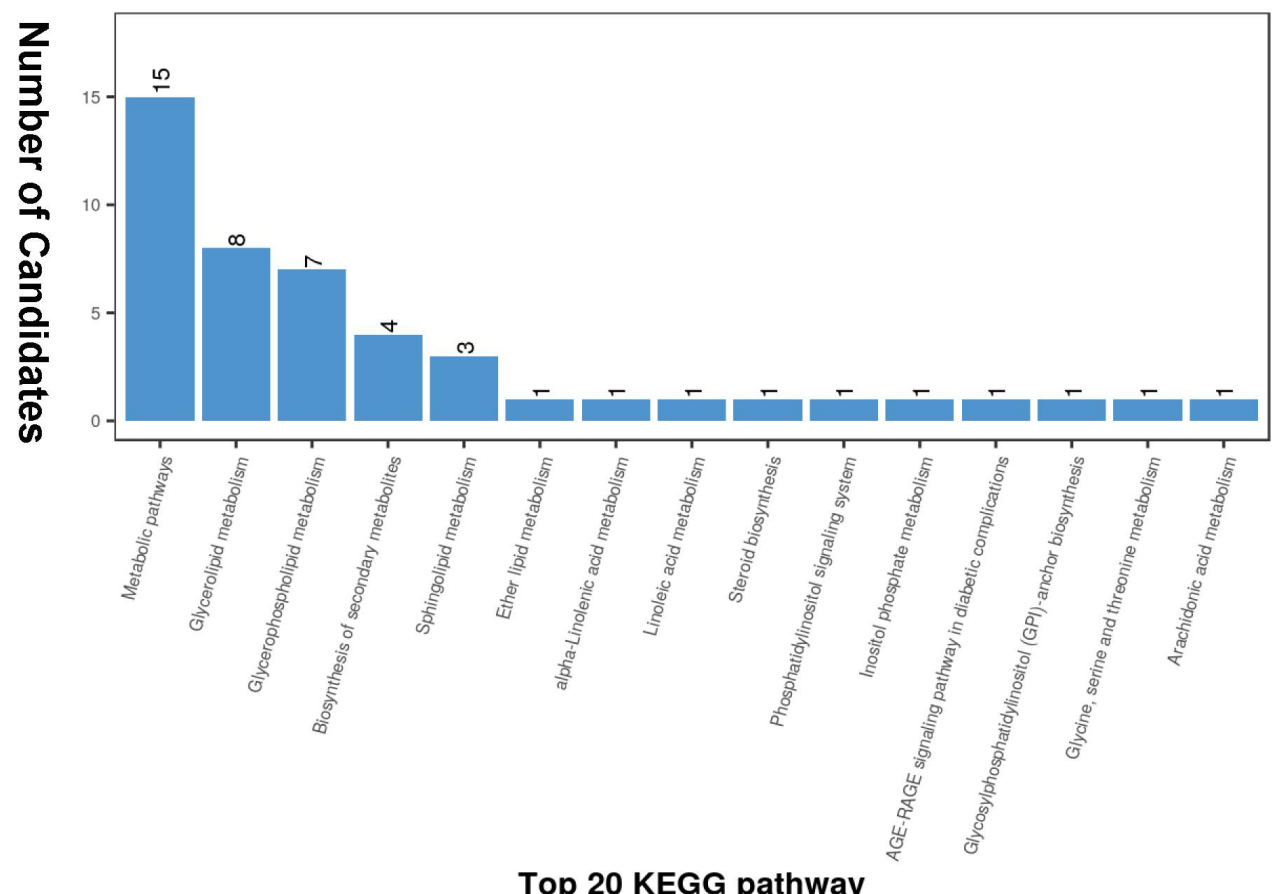

Top 20 KEGG pathway

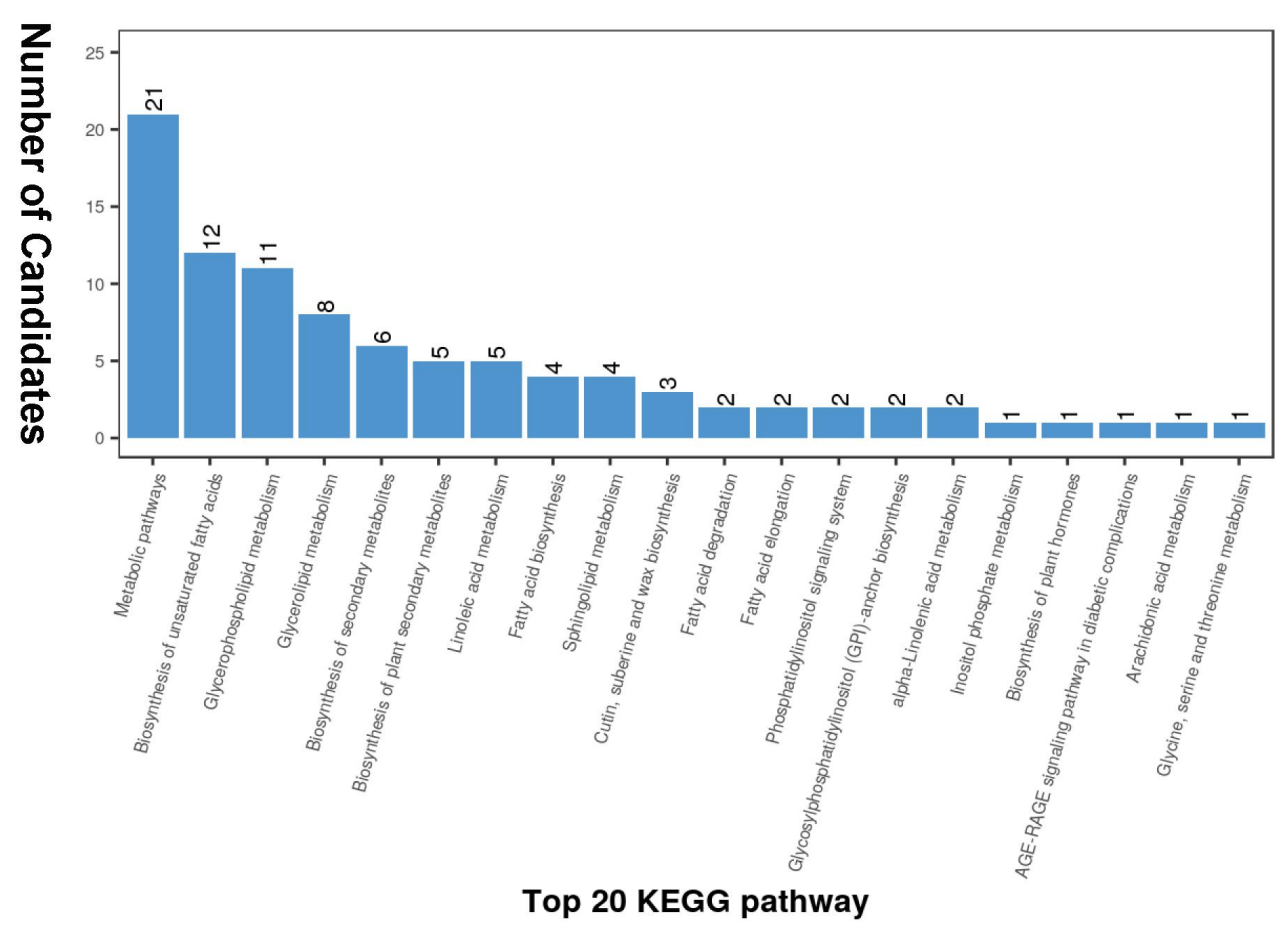

Figure S8. KEGG pathway enrichment analysis of 547 lipid features in secondary MS profile. A, KEGG pathway enrichment of 292 lipid species with positive model. B, KEGG enrichment analysis of 255 lipids with negative model. KEGG pathways fulfilling the criterion of a cut-off P-value $\leqslant 0.05$ were defined as significantly enriched. 

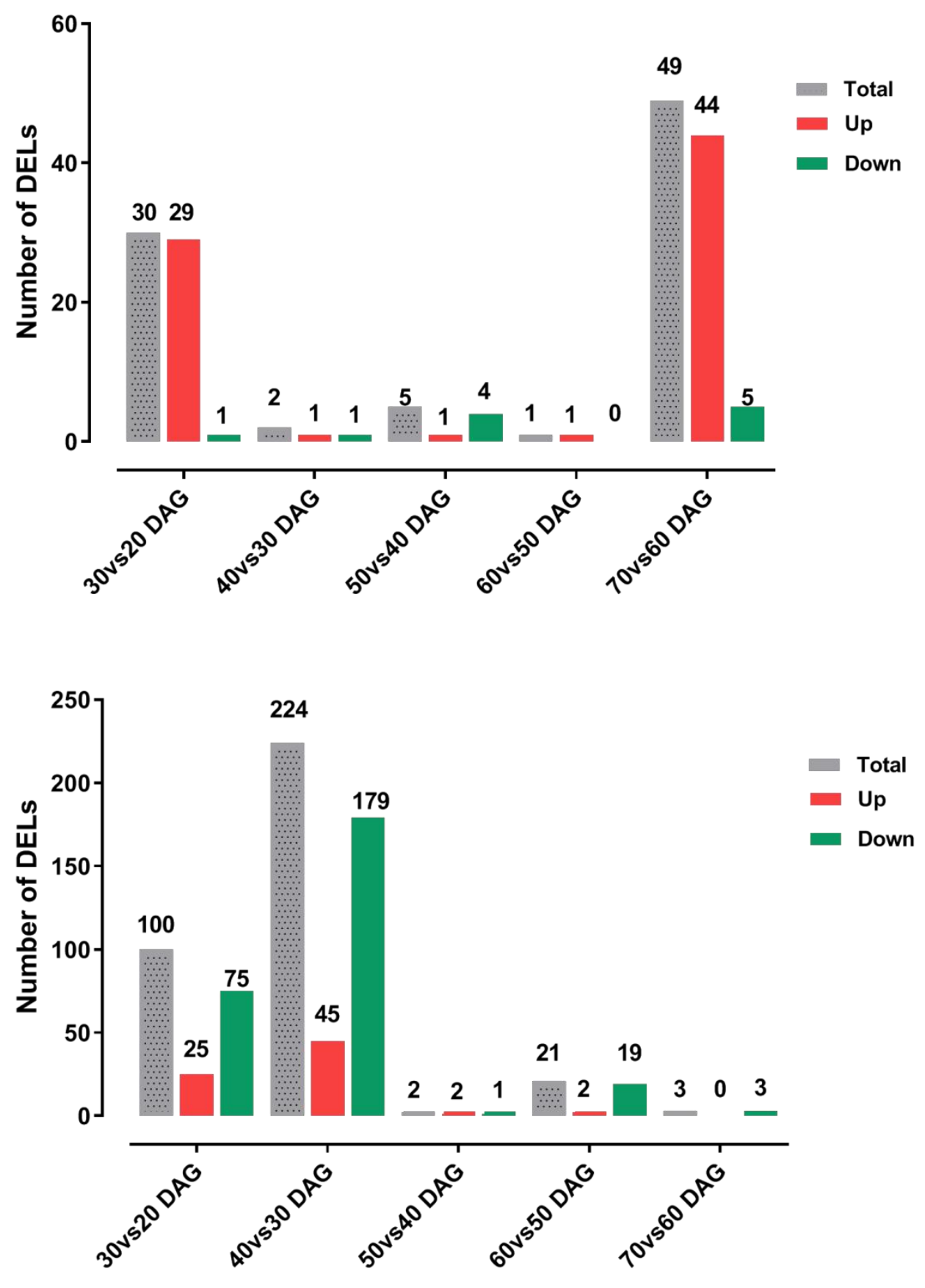

Figure S9. Statistical analysis of the number of DELs distribution in L70 and H176, respectively. A, The number of DELs distribution during the normal peanut seed development (Table S5). B, Statistical analysis of DELs' number at different seed developmental stages in H176 (Table S8). 


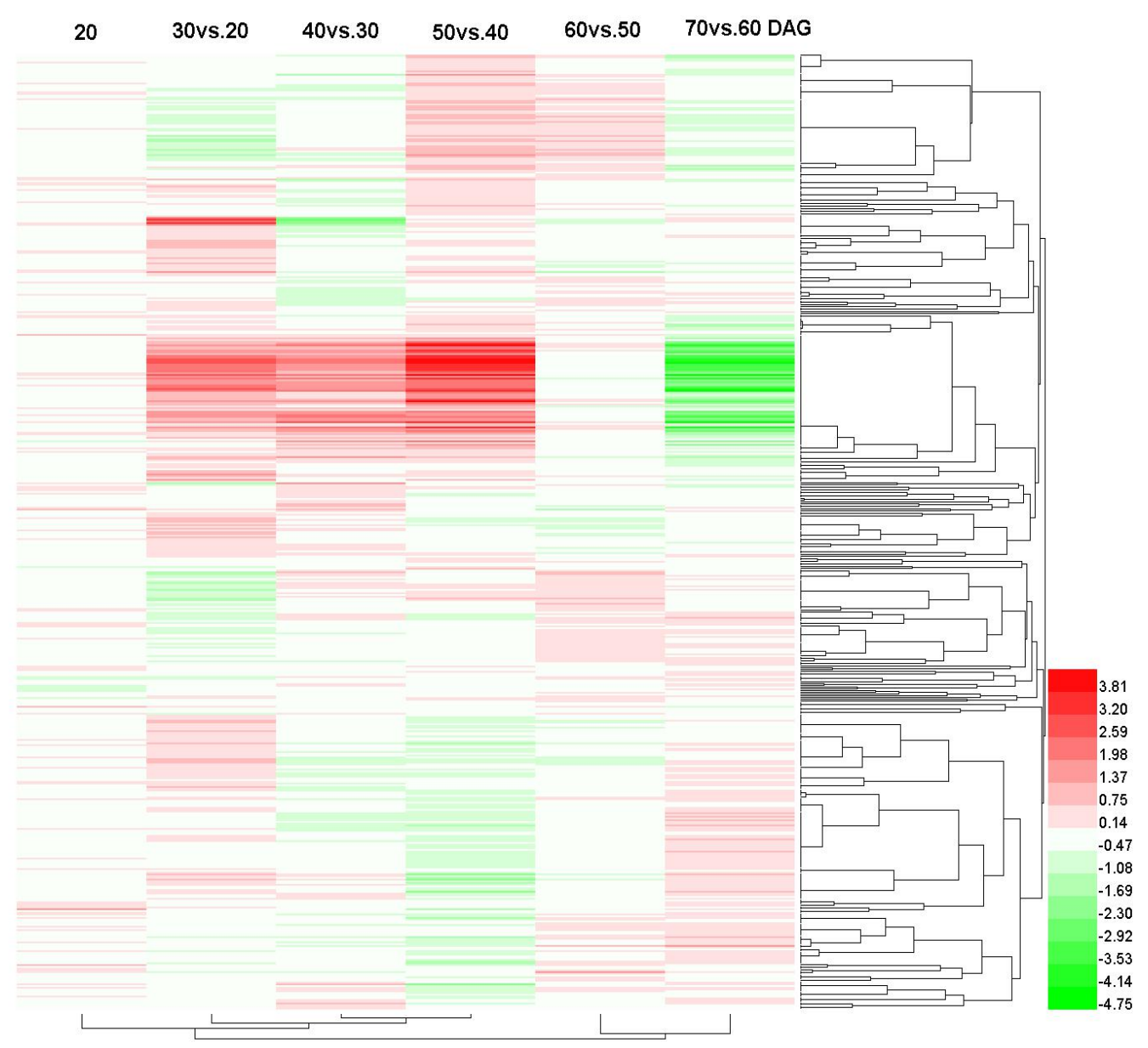

Figure S10. Heatmap (Table S7) displayed the variation trend of 547 lipids during the seed development in normal peanut variety (L70). 20 DAG represented the reference sample, each block represented the average relative intensity of lipid compound that came from statistics of three biological replicates (value of $\log ^{2}$ fold change), and the heatmap picture was generated by the software HemI. 


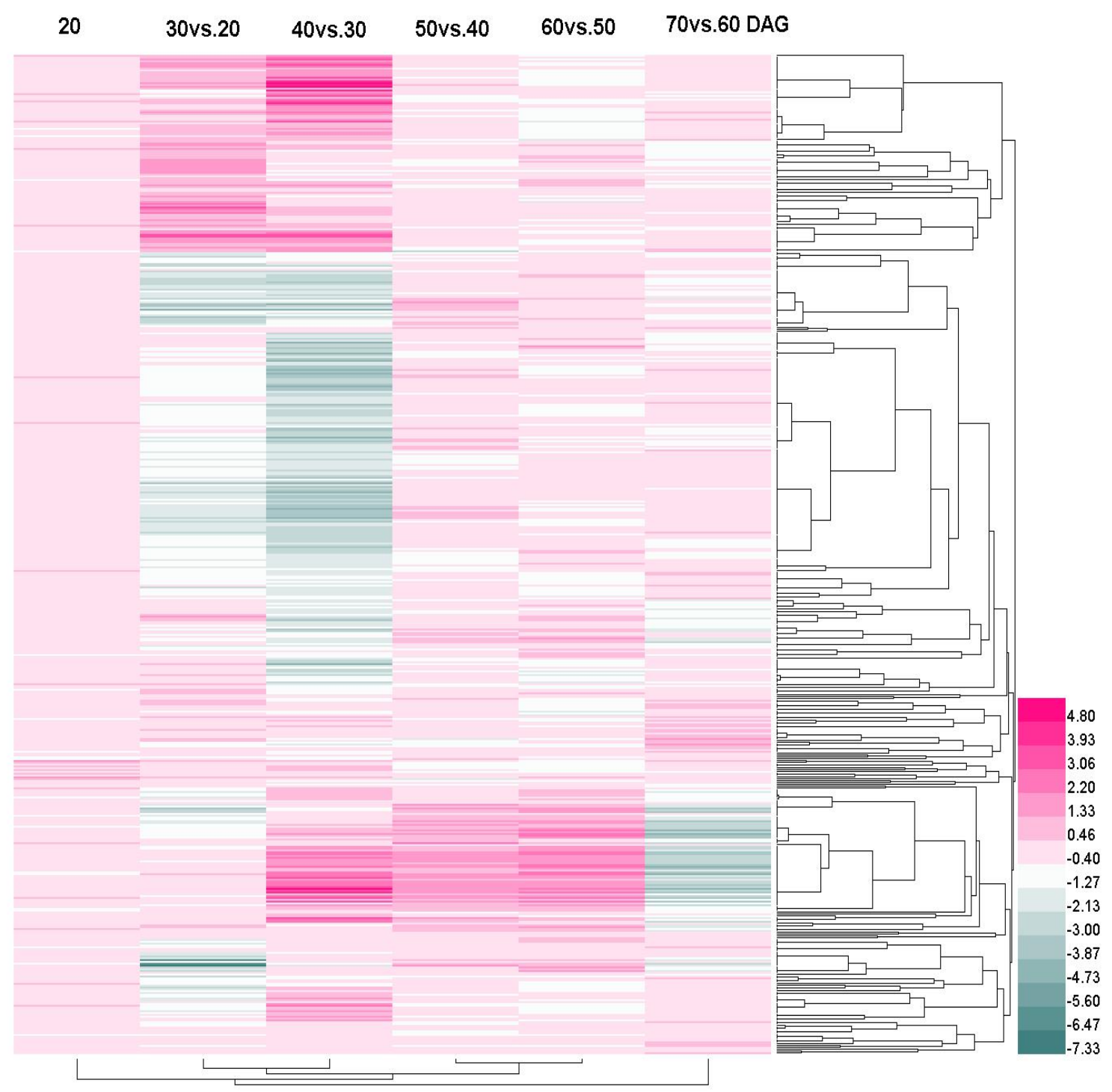

Figure S11. Heatmap (Table S10) showed the relative intensity of 547 lipids at each stage in high OA peanut (H176) seed. 20 DAG represented the reference sample, each block represented the average relative intensity of lipid compound that came from statistics of three biological replicates (value of $\log ^{2}$ fold change), and the heatmap picture was generated by the software HemI. 

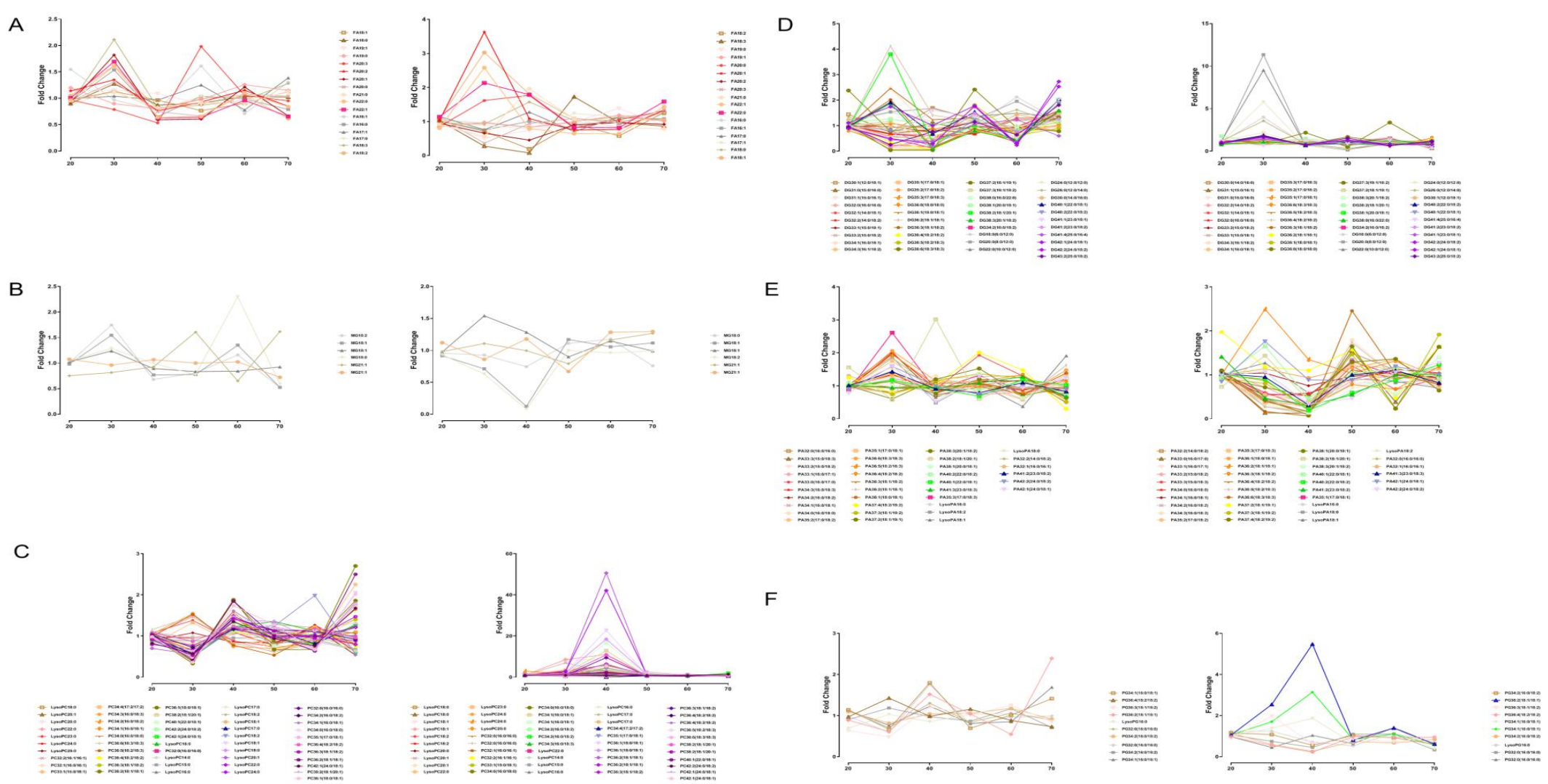

Figure S12. Variation tendency of lipid molecular species regarding of TAGs synthesis during the seed development in normal (L70) and high OA (H176) peanut, respectively. A-F represented the acyl FAs, MG, PC, DG, PA, and PG. These variation curves described by the average fold change in relative intensity of lipid molecular species (Table S6 and Table S9). 


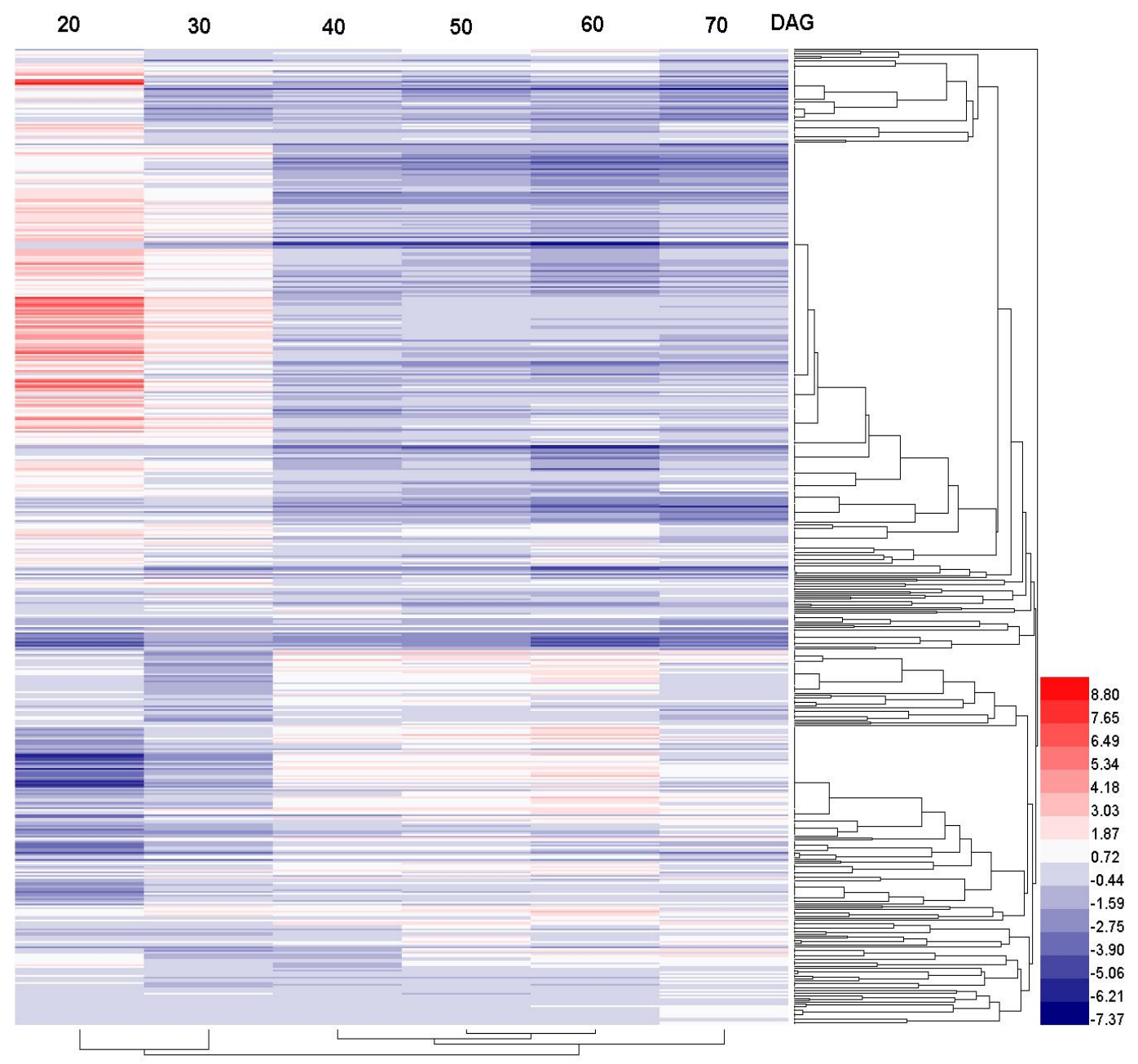

Figure S13. Heatmap (Table S11) showed the variation tendency of 547 lipids' relative intensity at each stage in the seed lipid expression profile of high OA (H176) vs. normal (L70) peanut. Each block represented the average relative intensity of lipid compound that came from statistics of three biological replicates (value of $\log ^{2}$ fold change), and the heatmap picture was generated by the software HemI. 

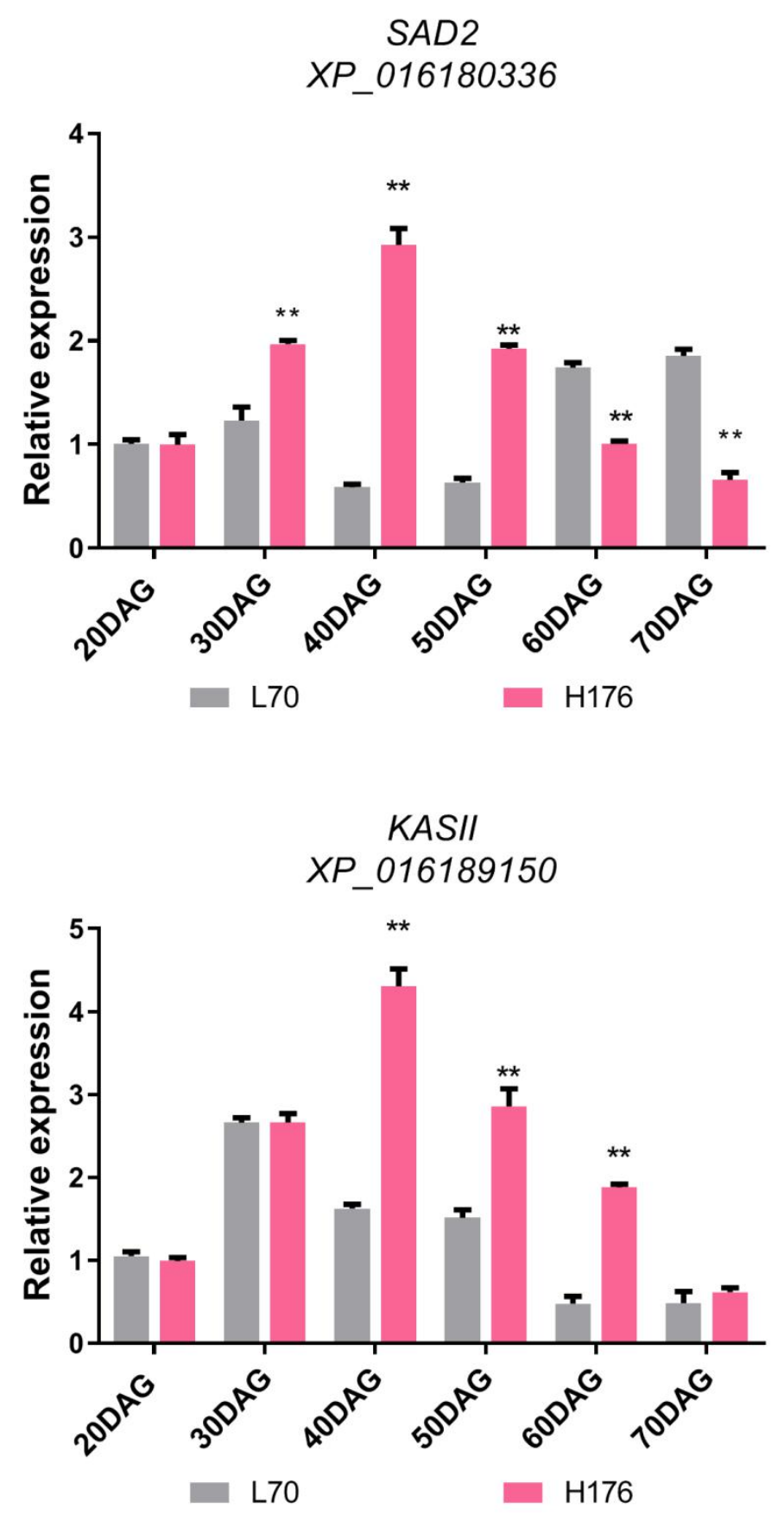

Figure S14. Quantitative real-time PCR validated the relative expression levels of $S A D 2$ and $K A S I I$ (Table 1) at the transcriptional level during the seed development in L70 and H176, respectively. Each measurement was carried out with three biological replicates, 20 DAG was used as the reference sample, and the values are shown as means $\pm \mathrm{SD}\left(* \mathrm{P}<0.05,{ }^{*} * \mathrm{P}<0.01\right)$ compared with normal cultivar $\mathrm{L} 70$. 
Profiles ordered based on the number of genes assigned
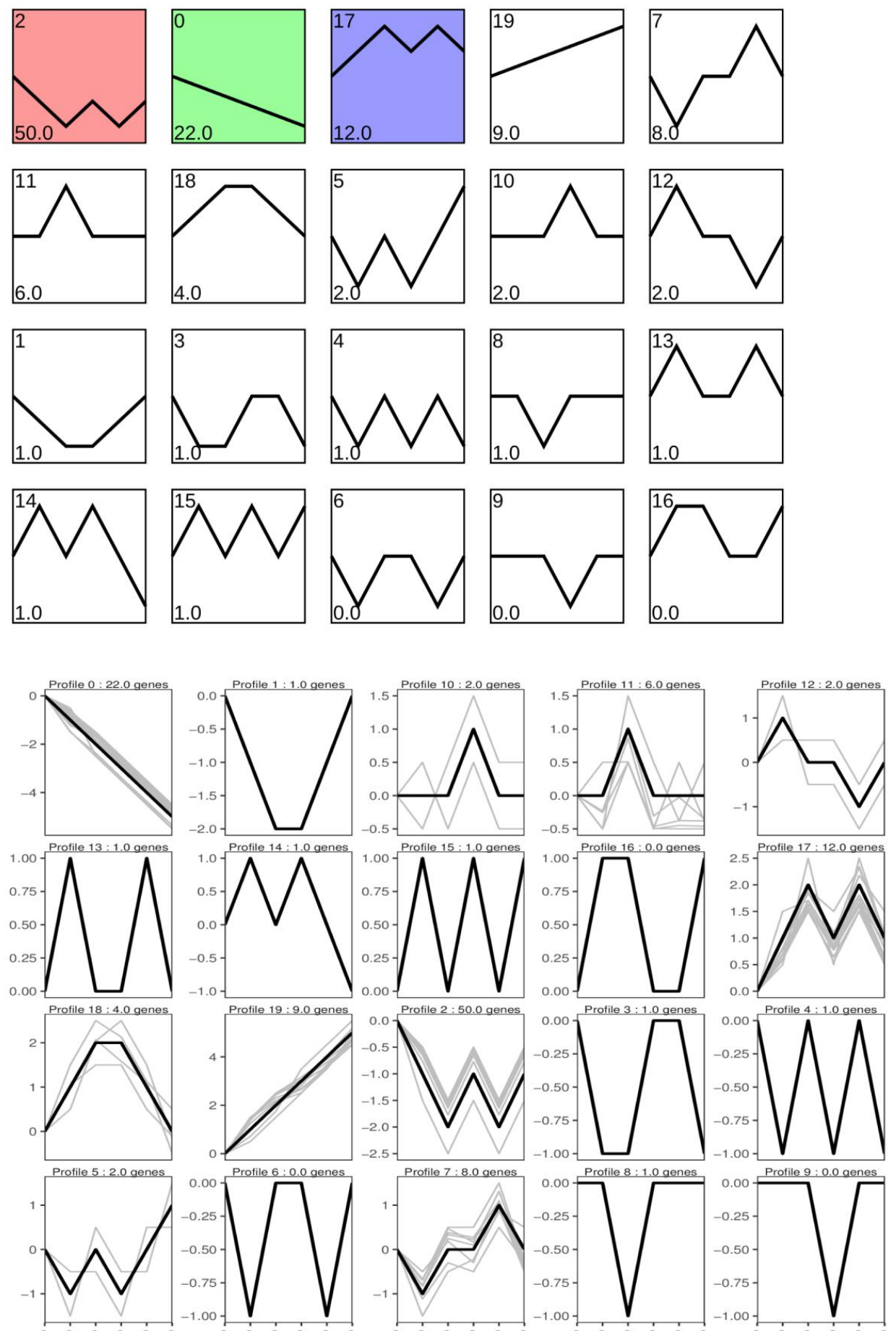

Figure S15. Trend analysis of DELs and DAPs expression profile (20 trends). Colored block trend: significant enrichment trend $(\mathrm{P}<0.05)$. Without color trend: the enrichment of significant trends (Table S17). 


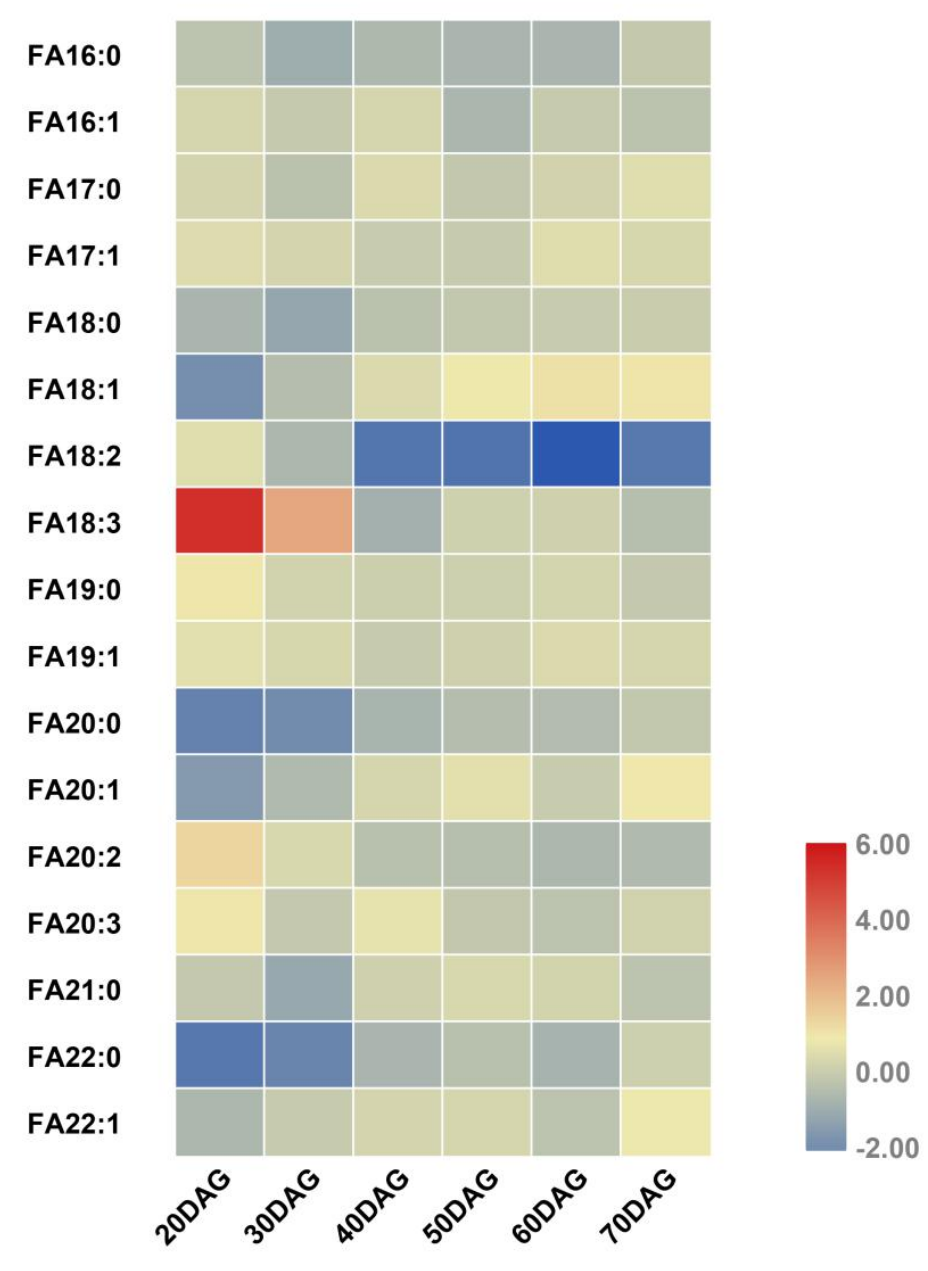

Figure S16. Heatmap showed the relative intensity variation of acyl FAs at each stage in the profile of H176 vs. L70. Each block represented the value of $\log ^{2}$ fold change (Table S18), and the heatmap picture was generated by the software TBtool. 

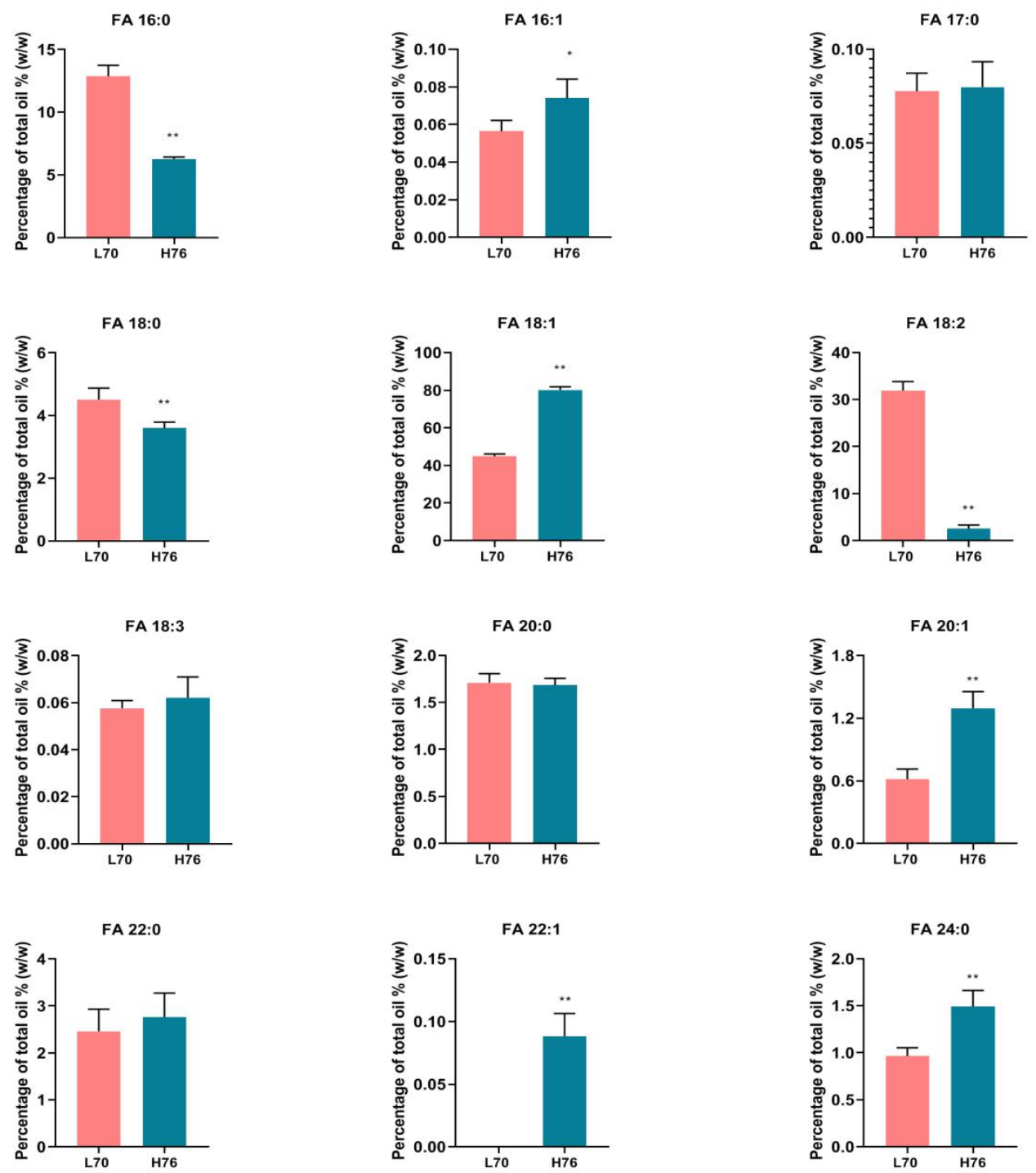

Figure S17. The composition of fatty acids in dehydrated peanut seeds (H176 and L70). Each measurement was carried out with four biological replicates, values are shown as means $\pm \mathrm{SD}(* \mathrm{P}<0.05, * * \mathrm{P}<0.01)$ compared with normal cultivar L70 (Table S19). w/w indicates each fatty acid weight compared with total oil weight. 